\title{
Off-plane gratings for Constellation-X
}

\author{
R. McEntaffer ${ }^{*}$, W. Cash ${ }^{\mathrm{a}}$, A. Shipley ${ }^{\mathrm{a}}$ \\ ${ }^{a}$ Center for Astrophysics and Space Astronomy, University of Colorado
}

\begin{abstract}
A reflection grating spectrometer featuring off-plane, radial groove gratings for the Constellation-X mission will be presented. Preliminary work shows that off-plane designs can significantly enhance the scientific capabilities of the mission baseline design. Resolutions up to $\lambda / \Delta \lambda \sim 5000$ can be achieved. The design accomplishes the mission goal for throughput and may also significantly reduce the assembly tolerances. Detailed raytracing and performance assessment of a strawman configuration are included.
\end{abstract}

Keywords: X-ray Spectroscopy, Constellation-X, Reflection Grating Spectrometer, off-plane mount, high spectral resolution

\section{INTRODUCTION}

The Constellation-X mission will provide an instrument with large effective collecting area and high spectral resolution relative to past and current $\mathrm{x}$-ray observatories. An overview of the mission and science goals is given by Tananbaum, et al. ${ }^{1}$, and on the web at http://constellation.gsfc.nasa.gov.

Briefly, the mission incorporates three instruments, all designed to perform high throughput spectroscopy of astronomical x-ray sources. For x-ray energies above $10 \mathrm{keV}$ there is a hard x-ray telescope (HXT) and from 0.1 to 10 $\mathrm{keV}$, there is a large foil optic telescope known as the SXT. The SXT has its light divided between two instruments - a calorimeter and a reflection grating spectrometer (RGS). The calorimeter is optimized for spectroscopy from 1 to 10 $\mathrm{keV}$, and the reflection gratings are optimized for the softest $\mathrm{x}$-rays, below $1 \mathrm{keV}$ where the performance of the calorimeter falls off.

The RGS will use dispersive spectroscopy to provide high resolution at the long wavelength end of the $\mathrm{x}$-ray spectrum. In order to achieve the high spectral resolution required of the Constellation-X mission we propose using a high density, radial groove grating array in the off-plane mount capable of achieving $\lambda / \Delta \lambda$ of 5000 .

As spectrograph resolution rises, so does the scientific return. The resolution, $\lambda / \Delta \lambda$, must be greater than 300 to unambiguously identify lines in the X-ray spectrum, and thus obtain important plasma diagnostics. Several properties of a gas can be determined at a resolution of a few hundred: radial velocity to within $1000 \mathrm{~km} / \mathrm{sec}$, densities of He-like ions, charge states, excitation mechanisms, etc. At resolutions of a few thousand one gains information on dielectronic satellite lines, radial velocities to within $100 \mathrm{~km} / \mathrm{sec}$ and thermal Doppler profiles of $10^{7} \mathrm{~K}$ gas.

The scientific goals that Constellation-X intends to achieve include:

-Mapping the geometry of the accretion disk around the central engine of an Active Galactic Nuclei (AGN)

-Measuring the spin of black holes using the relativistically broadened Fe Ka emission line from AGN

-Measuring the total luminosity of AGN and how this contributes to the total energy output of the Universe

-Determining galaxy cluster dynamics revealing effects of mergers

-Establishing abundances of intracluster gas as a function of time

-Revealing the baryon content of the Intergalactic Medium (IGM)

-Establishing abundances in the Interstellar Medium (ISM)

* randy@origins.colorado.edu; phone 1303 492-5835; fax 1303 492-5941; http://casa.colorado.edu; Center for Astrophysics and Space Astronomy, Astrophysics Research Laboratory, 593 UCB, $125538^{\text {th }}$ Street, Boulder, CO, USA 80309-0593 
Spectroscopy is provided by two instruments, a microcalorimeter and a Reflection Grating Spectrometer (RGS). Microcalorimeters have energy resolution elements, $\Delta E$, of constant size. Therefore, $\lambda / \Delta \lambda$ falls linearly with energy, dropping below requirements at energies less than $0.5 \mathrm{keV}$, a very rich part of the spectrum. Also, the microcalorimeter incorporates a long wavelength filter causing low efficiencies at these energies. Meanwhile, dispersive spectrometers have roughly constant wavelength bins so that $\lambda / \Delta \lambda$ increases as wavelength increases. Furthermore, efficiencies increase at these energies due to higher reflection efficiency which further combats the limitations of the microcalorimeter. This important point becomes even more crucial when the redshifts of objects cause the spectra to shift into softer energies. Therefore, by using this complimentary relation between microcalorimeters and dispersive optics it is possible to achieve high resolution over the entire bandpass, $0.25-40 \mathrm{keV}$.

\section{OFF-PLANE GRATING ARRAY}

\subsection{Off-plane geometry}

The off-plane mount at grazing incidence brings light onto the grating at a low graze angle, quasi-parallel to the direction of the grooves as shown in figure 1. The light is then diffracted through an arc, forming a cone, so that this mount is also known as conical diffraction ${ }^{2}$. The grating equation is now

$$
\sin \alpha+\sin \beta=\frac{n \lambda}{d \sin \gamma}
$$

where $\mathrm{d}$ is the spacing between grooves. $\gamma$ is the angle between the direction of the incoming ray and the direction of the groove at the point of impact. Light comes into the grating at an azimuthal angle of $\alpha$ along a cone with half-angle $\gamma$. It is then diffracted along the same cone of half-angle $\gamma$, but now with an azimuthal angle of $\beta$.

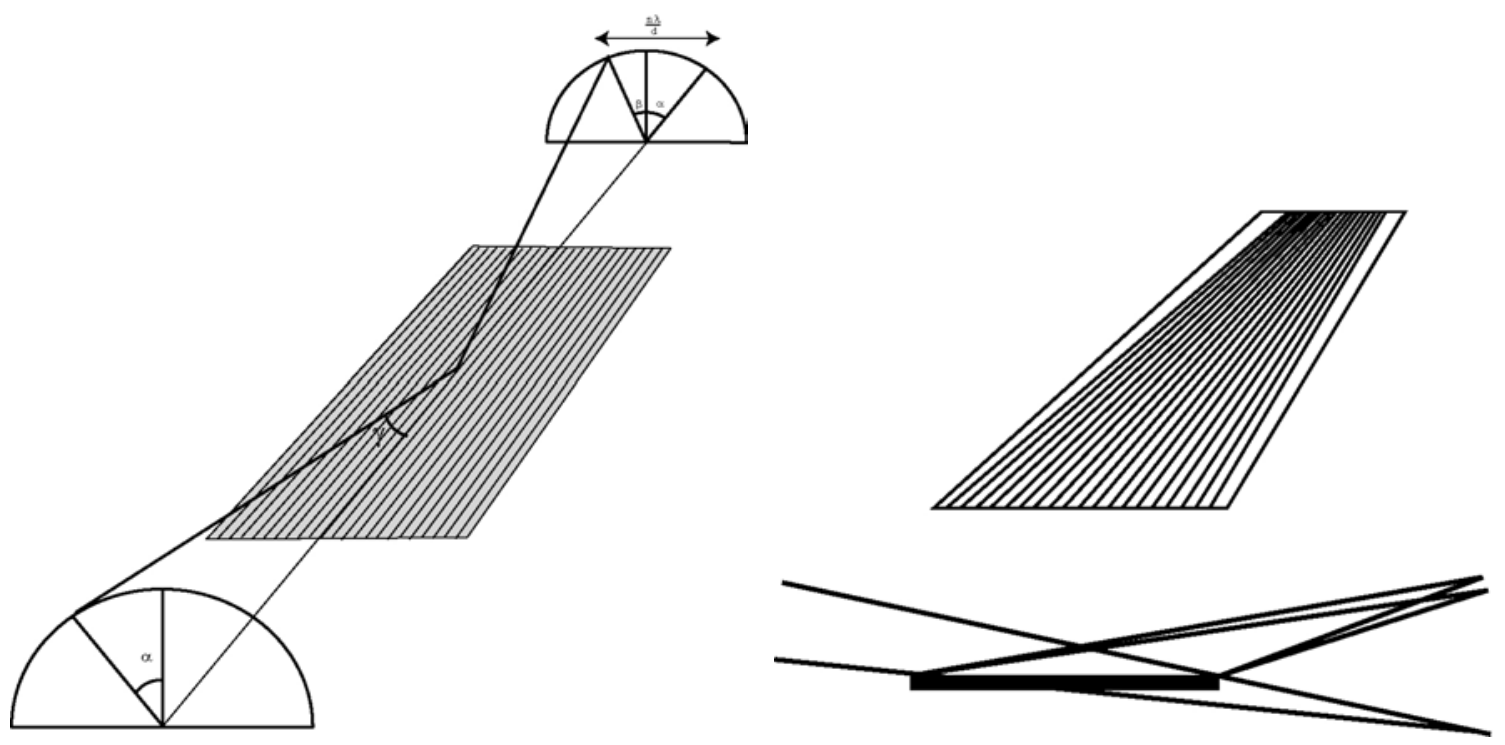

Fig. 1: Geometry of the off-plane mount. Quasi-parallel light at grazing incidence is diffracted in a cone around the direction of the rulings. Off-axis aberrations such as coma can be corrected using a radial groove grating in the converging beam.

When the gratings are placed behind the optics, the off-axis aberrations are corrected if the gratings compensate for the converging beam of the telescope. This can be done by using gratings that are ruled in a radial configuration where the grooves extend outward from a hub and match the convergence angle of the beam as shown in figure 1 .

The off-plane mount supplies the natural geometry for grazing incidence reflection gratings, thus offering several advantages. Among these advantages, effective area and resolution are key. 


\subsection{Effective area}

The effective diffraction efficiency of the off-plane mount can be substantially higher (often a factor of two) due to the groove illumination function ${ }^{4,5}$. In the off-plane mount the effect of groove shadowing is lessened. Furthermore, at graze angles around 2 degrees the theoretical efficiencies of gratings in the off-plane mount can easily reach $\geq 50 \%$. A model of the throughput for our off-plane design is displayed in figure 2 .

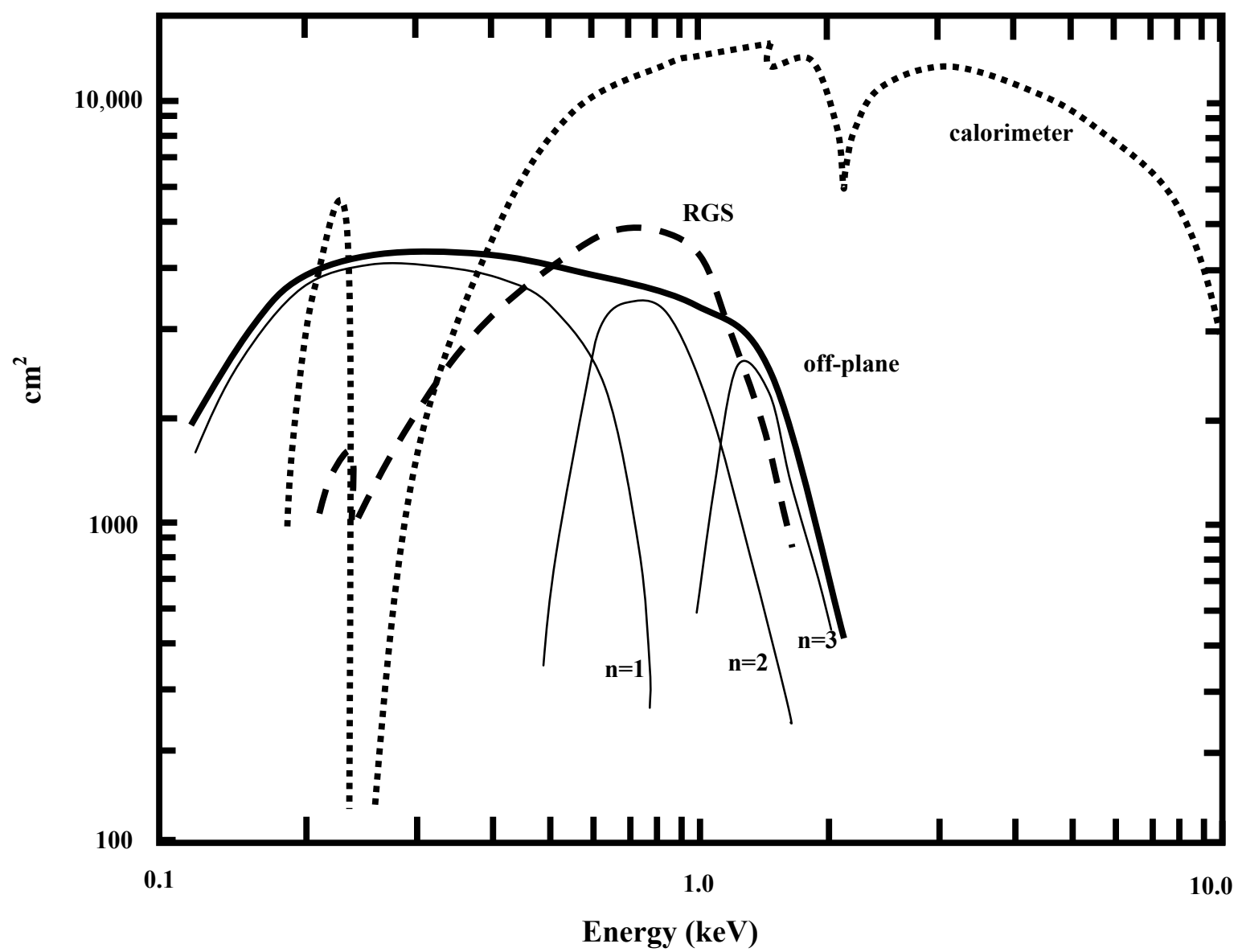

Fig. 2: Effective area as a function of energy. Dotted line: Calorimeter response. Dashed line: Constellation-X mission requirements for throughput. Thin solid lines: first, second and third order throughput for the off-plane grating array design. Bold solid line: Sum of orders for the off-plane mount.

As this figure shows, high throughput can be obtained from gratings in the off-plane mount. Also, this configuration allows the most efficient packing geometry for the grating array. These factors combine to achieve the required effective area for the mission. The telescope's total collecting area, $14,260 \mathrm{~cm}^{2}$, is available for use by the grating array, while the remainder must pass through to the calorimeter. Accounting for structural losses and CCD efficiency lowers this number to $\sim 11,000 \mathrm{~cm}^{2}$. The RGS has a goal of $5500 \mathrm{~cm}^{2}$ of effective area. Therefore, a grating array covering $62 \%$ of the telescope effective area with $50 \%$ efficient gratings (sum of orders) will be able to meet mission requirements. As seen by the Neviere efficiency curves, this can be achieved using the off-plane mount at grazing incidence. Also, gratings in the off-plane mount disperse into only a few orders allowing higher grating efficiencies for any one order, so that $50 \%$ efficiency is more easily obtained. 


\subsection{Resolution}

The most important advantage of the off-plane mount is the potential for high resolution. The diffracted light emerges at the same conical angle at which it approaches allowing dispersion through twice the graze angle ${ }^{2}$. Also, the light can be dispersed to high values of $\beta$ to achieve extra resolution as in an echelle spectrograph ${ }^{6}$. Furthermore, the higher effective diffraction efficiency due to efficient groove illumination, as previously discussed, also increases resolution. Resolution of a spectrograph can be written as

$$
R=\frac{(\sin \alpha+\sin \beta) \sin \gamma}{B \cos \alpha}
$$

where $B$ is the telescope spatial resolution in radians. At graze angles around 2 degrees and telescope resolution of 15 arseconds, typical resolutions are in the range between a few hundred and a thousand.

Sub-aperturing, a technique described fully by Cash $(1987,1991)^{2,7}$, can improve upon this resolution even further. The spatial resolution of X-ray optics is limited by scatter and surface figure errors, which are responsible for the $r^{-1.5}$ distribution in the point spread function. However, for a Wolter telescope with nested parabola/hyperbola mirrors the blur is not random but highly structured ${ }^{7}$. In fact, only certain mirrors contribute to a certain spot at the focal plane. Only when all mirrors are considered is the full image realized. Therefore, by stopping down the aperture the telescope resolution can be made to increase by limiting the spot size. As a result, the spectral resolution increases as shown in equation 2. For example, at a graze angle of $\gamma$, if the open aperture is stopped down to include only $\gamma$ radians of the $2 \pi$ radians in the annulus, then the resulting image is $1 / \gamma$ times narrower in that dimension, thus increasing the resolution. The implementation of sub-apertures for Constellation- $\mathrm{X}$ is shown in figure 3 .

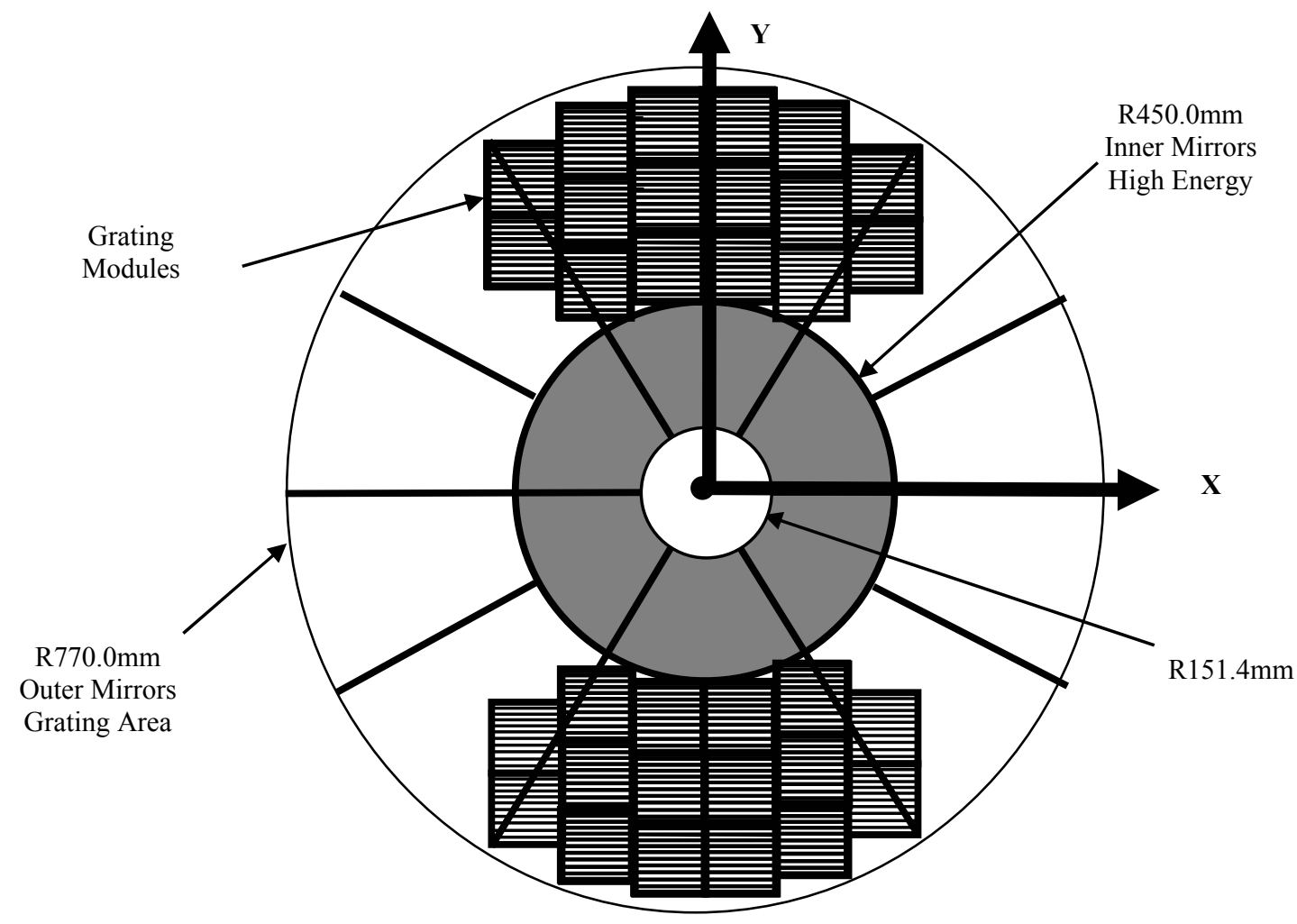

Fig. 3: Positioning of the grating array with respect to the telescope. This displays the technique of sub-aperturing. By only allowing mirrors close to the $\mathrm{Y}$ axis to illuminate the grating array leads to better spectral resolution. 
This schematic shows that the grating modules (figure 4) that constitute the grating array are placed predominantly in the $\mathrm{Y}$ direction. This is equivalent to stopping down the aperture in the $\mathrm{X}$ dimension, which corresponds to the dispersion direction. Therefore, the telescope resolution is improved in the dispersion direction, which improves spectral resolution.

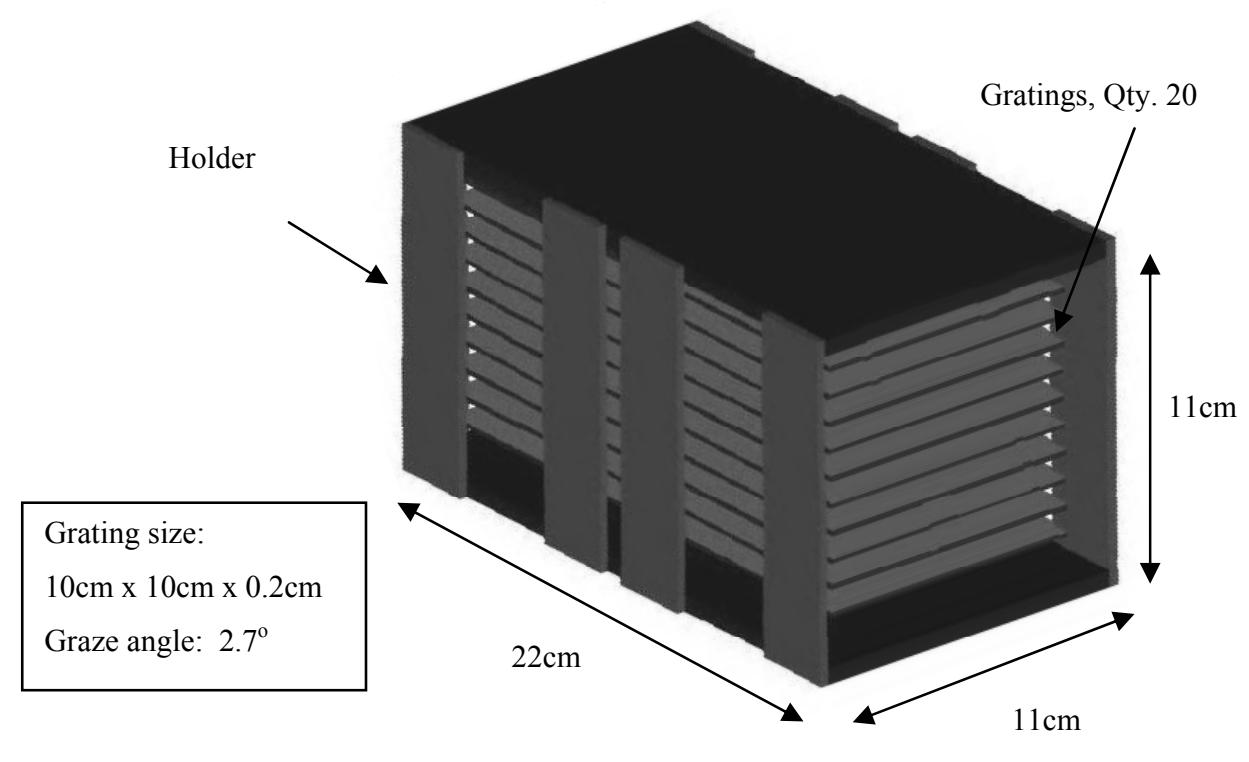

Fig 4: One off-plane grating module.

\subsection{Other advantages}

Fabrication tolerances are relaxed for off-plane mounts. Optical errors in fabrication cause blur in the in-plane direction. Off-plane mounts disperse perpendicular to this direction so that this fabrication error does not affect resolution.

\section{OFF-PLANE GRATING ARRAY RAYTRACE}

A design for the RGS utilizing a grating array in the off-plane mount has been raytraced. The gratings have a radial groove configuration with 5800 grooves $/ \mathrm{mm}$ spacing at the top. Each is $100 \mathrm{~mm}$ x $100 \mathrm{~mm}$ with a $9.3 \mathrm{~m}$ throw. The resulting spectral lines are shown in figure 5. The figure is a sequence over wavelength with each plot showing two lines separated by $0.028 \AA$. The raytrace consisted of 5 grating modules. These modules were placed side-by-side along the dispersion direction. The resolution ranges from $\sim 700$ to $\sim 6000$ over this bandpass as displayed in figure 6 . From figure 5 it is evident that the spectral line shape is dependent on wavelength. This is the reason that the resolution curve in figure 6 falls off faster than theoretical. This plot shows how the microcalorimeter resolution falls off at low energy and how the RGS makes up for it. The dotted lines show the baseline resolution required for the mission. The bold, solid lines show the resolution obtained from the raytrace of the off-plane grating array in first, second and third order. Finally, the dashed lines display the decreased resolution obtained from a raytrace of the off-plane design with looser figure tolerances on the gratings. 


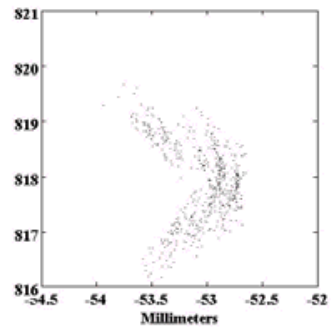

$10 \AA$

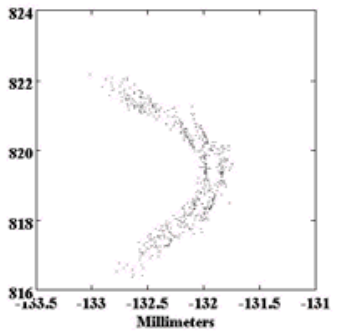

$25 \AA$

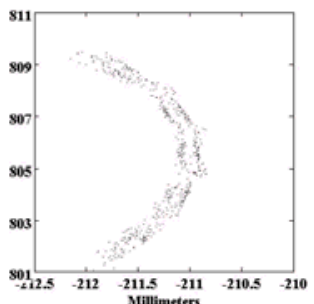

$40 \AA$

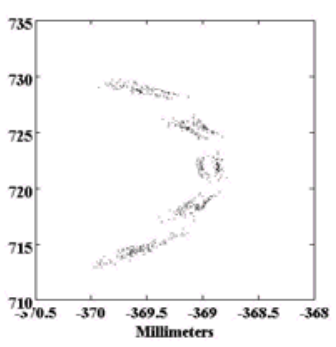

$70 \AA$

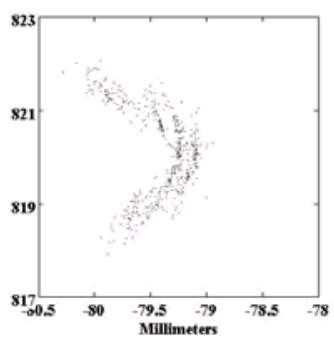

$15 \AA$

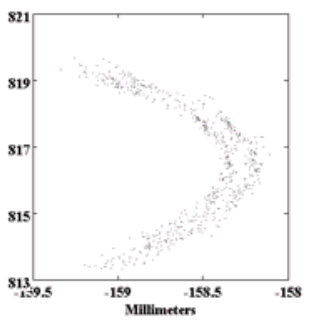

$30 \AA$

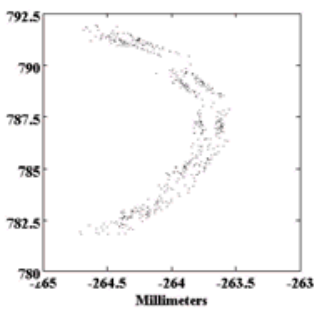

$50 \AA$

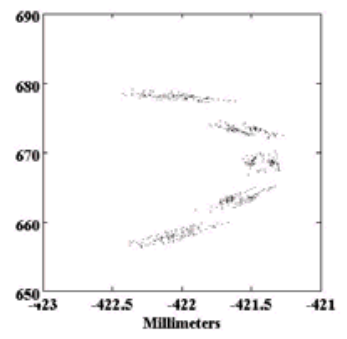

$80 \AA$

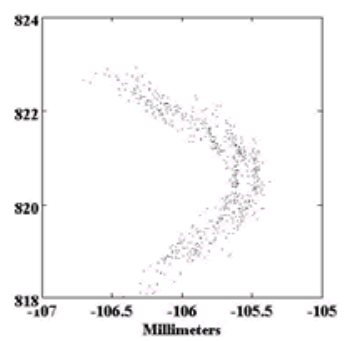

$20 \AA$

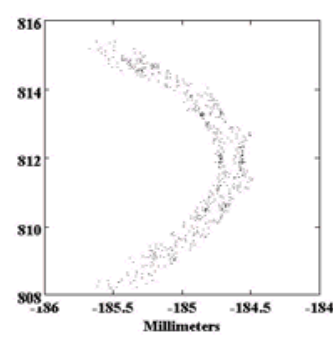

$35 \AA$

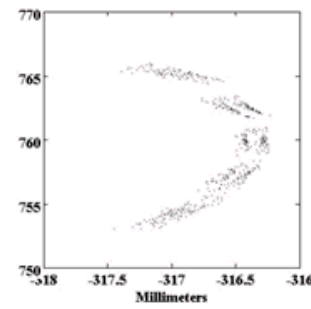

$60 \AA$

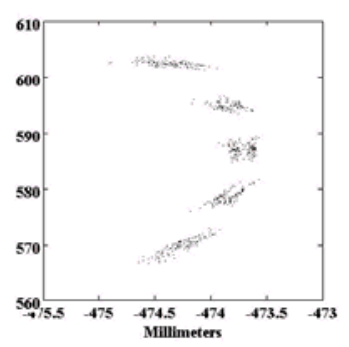

$90 \AA$

Fig. 5: Raytrace of wavelength pairs, $\lambda$ and $\lambda+0.028 \AA$. 


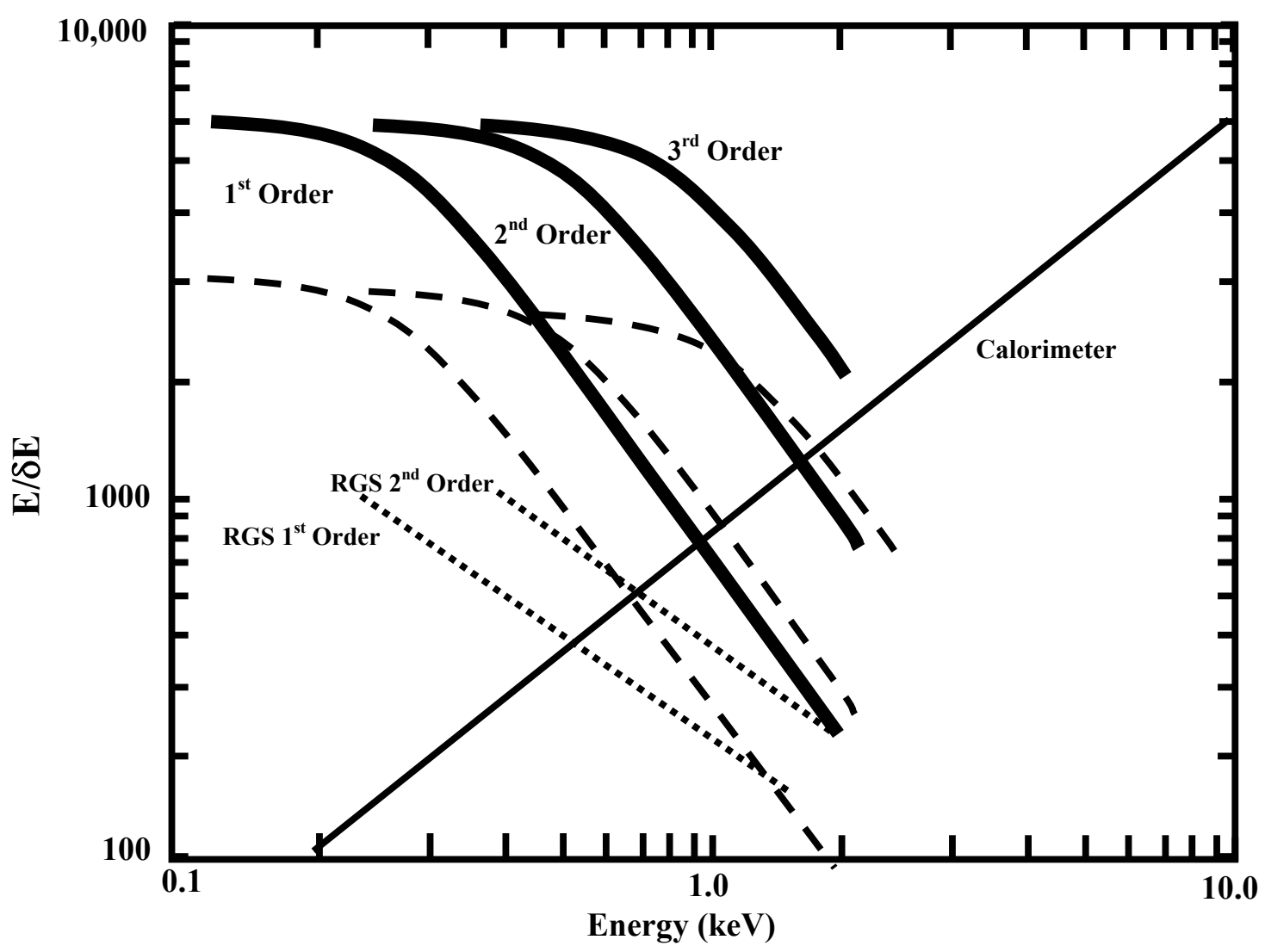

Fig. 6: Resolution as a function of energy. The thin solid line exhibits the microcalorimeter response. The dashed lines display the mission requirements at first and second order. The bold solid lines show the resolution obtained from our raytrace while the dashed lines are indicative of our design with looser figure tolerances on the gratings.

\section{DISADVANTAGES}

One of the main disadvantages of the off-plane grating array is the requirement of high groove density. However, the state-of-the-art in diffraction gratings can provide the needed densities. Gratings are being made with 6000 grooves $/ \mathrm{mm}$ and are currently being used on FUSE and COS.

The other drawback to using the off-plane mount is the problem with the depth of focus. However, it's important to note that this only becomes an issue when attempting to achieve resolutions greater than 1200 . At lower resolutions the blur due to differing depths of focus is negligible compared to the spot size. This becomes a problem because the detector is laterally removed from the focus of the telescope. Therefore, light originating from one side of the telescope has a longer optical path distance to achieve the same focus than light on the opposite side. We are studying ways of resolving this issue. Curving the gratings and varying their radii of curvature as a function of their position would present a solution, but would also significantly increase the difficulty of fabrication. The easiest solution seems to be modifying the telescope. These modifications need only apply to the segments supplying light to the grating array. If the azimuthal radii of curvature of the parabola/hyperbola pairs are varied as a function of their position then the light will focus at the same point over the entire array. For instance, if the radius of curvature slightly increases for each parabola/hyperbola as the distance from the detector increases, then the focal lengths increase as well to compensate for the detector's position, thus giving a perfect focus. 
One factor that may limit resolution is the 5 arcsecond aspect solution. The telescope pointing is not known to better than 5 arcseconds. However, if a 0 order monitor is incorporated then the higher resolution can be retained.

\section{GRATING FABRICATION}

The largest fabrication issue will be obtaining high groove density. Currently, Jobin-Yvon is under contract to provide a test grating that is holographically ruled and blazed with radial grooves within the next year. Holographic ruling will allow the gratings to achieve efficiencies that a conventionally machined grating would not. This process will utilize the state of the art to achieve groove densities as high as 5800 grooves $/ \mathrm{mm}$. Also, we are investigating mass reducing remedies such as replication of the gratings on thin, light substrates including glass, aluminum and silicon.

\section{CONCLUSION}

A RGS incorporating an array of high-density, radial groove reflection gratings in the off-plane mount will be able to achieve Constellation-X mission requirements for effective area and resolution. The configuration allows for maximum packing efficiency and therefore, maximum effective area. Raytraces of a strawman configuration have shown that resolutions of $>5000$ can be achieved. Fabrication errors do not decrease resolution and test gratings are currently being made. The off-plane model for the RGS provides a simple and robust design for achieving mission goals and may even expand on these.

Resolutions in the thousands will increase the scientific capabilities of the observatory. With higher resolution, Constellation-X will be able to probe to even smaller radii in the accretion disk of an AGN, establish dynamics of a variety of hot media to a finer detail and determine line profiles with much more accuracy thus constraining the characteristics of the gas even further.

\section{ACKNOWLEDGMENTS}

This work was supported by NASA Grant NAG5-11850. RM acknowledges the support of the NASA Graduate Student Research Program.

\section{REFERENCES}

1. H.D. Tananbaum, N.E. White, J. A. Bookbinder, F. E. Marshall, and F. Cordova, "Constellation Xray mission implementation concept and science overview," Proc. Soc. Photo-Opt. Instr. Eng., 3765, 62-72, 1999.

2. W. Cash, "X-ray optics. 2: A technique for high resolution”, Applied Optics, 30, 1749-1759, 1991.

3. R. Catura, R. Stern, W. Cash, D. Windt, J.L. Culhane, J. Lappington, K. Barnsdale, "X-ray Objective Grating Spectrograph”, Proc. Soc. Photo-Opt. Instr. Eng., 830, 204-216, 1988.

4. M. Neviere, D. Maystre, W.R. Hunter, "On the use of classical and conical diffraction mountings for XUV gratings", JOSA, 68, 1106-1113, 1978.

5. W. Werner, "X-ray efficiencies of blazed gratings in extreme off-plane mountings", Applied Optics, 16, 2078-2080, 1977.

6. W. Cash, "Echelle spectrographs at grazing incidence", Applied Optics, 21, 17, 1982.

7. W. Cash, "X-ray optics: a technique for high resolution imaging", Applied Optics, 26, 2915-2920, 1987. 